\title{
Microscopic Studies of Liver and Kidney in Mice Exposed to Silver Nanoparticles
}

\author{
Raquel Marçal ${ }^{1}$, Joana Carrola ${ }^{2}$, Ivana Jarak ${ }^{2}$, M. Luisa Corvo ${ }^{3}$, Iola F. Duarte ${ }^{2}$ and Maria de Lourdes \\ Pereira $^{4}$ \\ ${ }^{1 .}$ Departamento de Biologia, Universidade de Aveiro, Portugal. \\ 2. CICECO - Aveiro Institute of Materials, Departamento de Química, Universidade de Aveiro, Portugal. \\ ${ }^{3}$ Med.ULisboa, Departamento de Farmácia Galénica e Tecnologia Farmacêutica, Faculdade de \\ Farmácia, Universidade de Lisboa, Lisboa, Portugal. \\ 4. CICECO - Aveiro Institute of Materials, Departamento de Biologia, Universidade de Aveiro, Portugal.
}

Silver nanoparticles (AgNPs) are widely used in different consumer and medical products due to their strong broad-spectrum antimicrobial activity $[1,2]$. Indeed, these NPs have applications in various commercially available products for wound healing, implantable medical devices, biosensors, as well as in emerging fields such as anticancer agents. However, despite their promising potential, increasing evidence suggests possible adverse effects of AgNPs in experimental animals, as well as human health (2). Our previous studies have shown that Ag-NPs, at a sublethal dose, disturbed cellular and systemic metabolism, largely affecting the pathways involved in energy production and antioxidant protection [3]. Hepatic and renal toxicity was also reported in mice exposed to AgNPs and authors underlined the role of oxidative stress as the major mechanism responsible for the toxic effects [4-5]. The current study aimed to explore the histological effects of AgNPs on mice liver and kidney.

Male mice (2 months old) were randomly divided into three groups, a control group ( $\mathrm{n}=5)$ and two experimental groups $(\mathrm{n}=5$ each) i.v. administered with $30 \mathrm{~nm}$ polyethyleneglycol (PEG)-coated AgNPs (8 $\mathrm{mg} / \mathrm{kg}$ of body weight) and euthanized at 24 and 48 hours post-injection. Blood, liver, spleen, lungs, kidneys, heart and muscle were collected and kept in a $10 \%$ (v/v) neutral buffered formalin solution for histological studies. For this study some hepatic and renal tissues fragments were processed for histology and results are presented. Samples were dehydrated and included in paraffin wax. Sections 4-5 $\mu$ m thick were stained with haematoxylin and eosin (HE) for light microscopy. Observations and micrographs were conducted on a digital camera (Olympus Camedia C-5060) coupled to a microscope (Olympus BX41, Tokyo, Japan). Animal tests were conducted agreeing to ethical national guidelines for animal experimentation.

A survival rate of $100 \%$ was observed. No significant differences on general behavior were observed between control mice and those in any of the AgNP exposure groups. Hepatic and renal sections from animals of the control group evidenced normal regular features (Fig. 1A, D). Several degenerative lesions were noted in the hepatic tissue of Ag-NPs exposed animals of both $24 \mathrm{~h}$ and $48 \mathrm{~h}$ assays, being more expressive at 24h (Fig.1B). After 24h of exposure to AgNPs suspensions no microscopic changes were noted on renal sections. However, histological results showed degenerative changes within the glomerulus of $48 \mathrm{~h}$ exposed mice (Fig.1E). In addition, the lumina of most of the tubules were closed by sloughing of epithelial cells (Fig.1F). Based on our data of mild hepatic, and renal tubular injury after AgNP exposure this fact may compromise the functional activity. Further studies of AgNPs are being conducted in mice for a better understanding of the pathway mechanisms underlying those lesions [6].

References:

[1] R. Y. Pelgrift and A. J. Friedman, Adv. Drug Del. Rev. 65 (2013) p. 1803.

[2] L. Wei et al., Drug Discov. Today 20:5 (2015) p. 595.

[3] J. Carrola et al., NanoPT2015, 11-13 Feb., Porto, Portugal (2015) p. 32. 
[4] R. Shrivastava et al., Toxicol. Ind. Health (2014) doi: 10.1177/0748233714562623.

[5] M.M. El Mahdy et al., Exp. Toxicol. Pathol. 6:1 (2015) p. 21.

[6] This work was developed in the scope of the project CICECO-Aveiro Institute of Materials (Ref. FCT UID/CTM/50011/2013), financed by national funds through the FCT/MEC and when applicable co-financed by FEDER under the PT2020 Partnership Agreement. Funding by FEDER through COMPETE and by FCT, under the project FCOMP-01-0124-FEDER-021456 (Ref. FCT PTDC/SAU-TOX/120953/2010) and grant $\mathrm{SFRH} / \mathrm{BD} / 79494 / 2011$, is acknowledged.
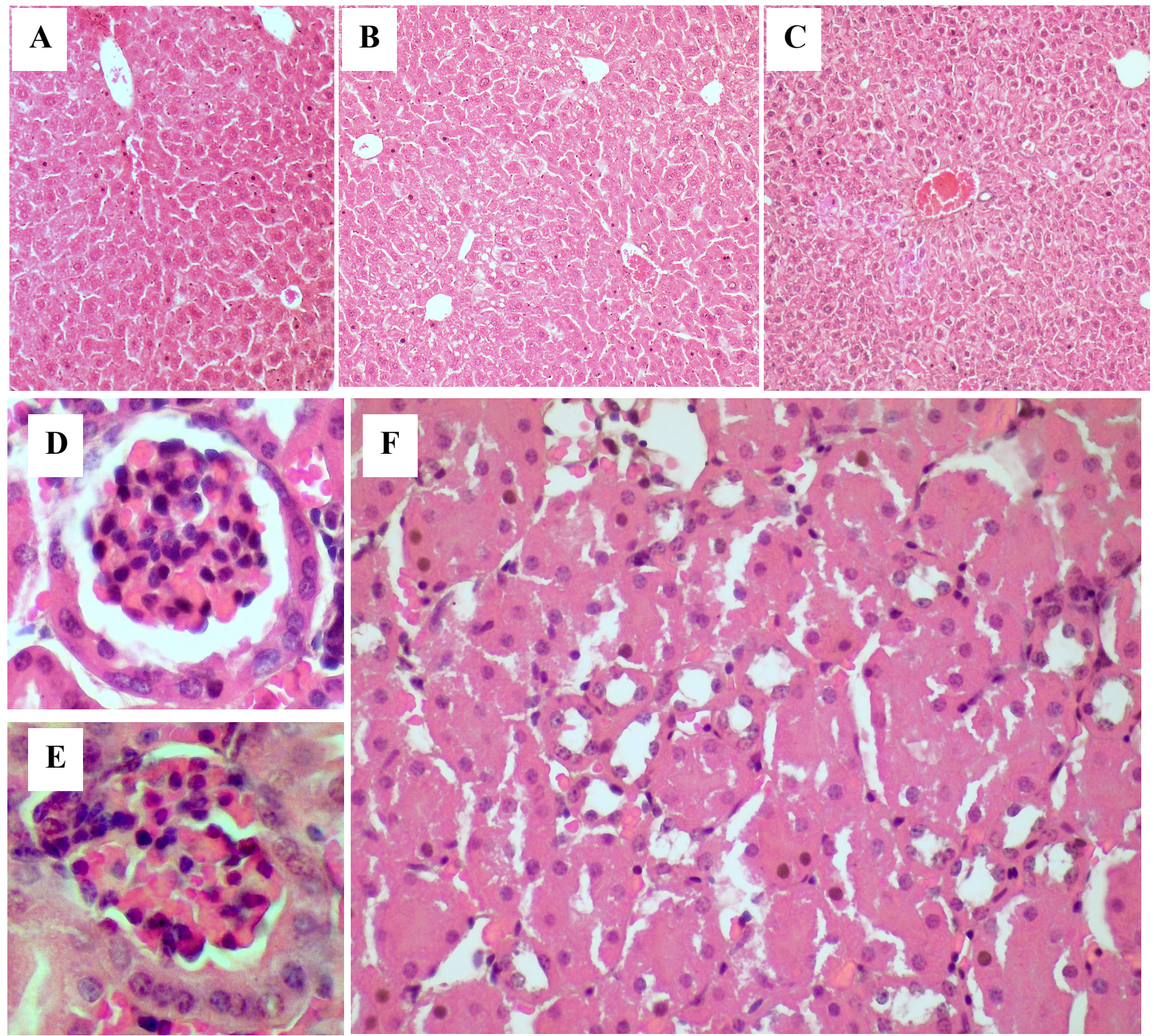

Figure 1. Representative light microphotographs of HE staining of hepatic and renal sections (A-F): A control liver; B-C: Ag-NPs-exposed animals during 24h and 48h, respectively; vacuolation is noted on B; D - Glomerulus from a control mouse; E - Glomerulus from Ag-NPs-exposed mouse for 48h showing dilatation of Bowman's capsule, although the capillary network displays regular features. F Obstruction of lumen within renal tubules. A- C-400x; D, E-800x; F-600x. 